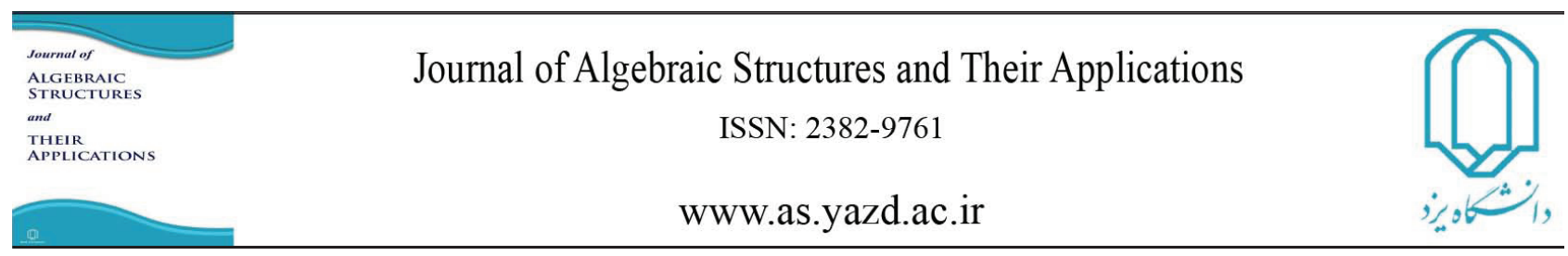

Algebraic Structures and Their Applications Vol. 4 No. 1 ( 2017 ) pp 53-58.

\title{
D-SPECTRUM AND D-ENERGY OF COMPLEMENTS OF ITERATED LINE GRAPHS OF REGULAR GRAPHS
}

\author{
GOPALAPILLAI INDULAL*
}

\author{
Communicated by S. Alikhani
}

\begin{abstract}
The $D-$ eigenvalues $\left\{\mu_{1}, \mu_{2}, \ldots, \mu_{p}\right\}$ of a graph $G$ are the eigenvalues of its distance matrix $D$ and form its $D$ - spectrum. The $D$ - energy, $E_{D}(G)$ of $G$ is given by $E_{D}(G)=\sum_{i=1}^{p}\left|\mu_{i}\right|$. Two non cospectral graphs with respect to $D$ are said to be $D-$ equi energetic if they have the same $D$ - energy. In this paper we show that if $G$ is an $r$ - regular graph on $p$ vertices with $2 r \leq p-1$, then the complements of iterated line graphs of $G$ are of diameter 2 and that $E_{D}\left(\overline{L^{k}(G)}\right), k \geq 2$ depends only on $p$ and $r$. This result leads to the construction of regular $D$ - equi energetic pair of graphs.
\end{abstract}

\section{INTRODUCTION}

Let $\mathrm{G}$ be a connected graph with vertex set $V(G)=\left\{v_{1}, v_{2}, \ldots, v_{p}\right\}$ and size (= number of edges) $q$. The distance matrix $D=D(G)$ of $G$ is defined so that its $(i, j)$ - entry is equal to $d_{G}\left(v_{i}, v_{j}\right)$, the distance (= length of the shortest path [3]) between the vertices $v_{i}$ and $v_{j}$ of $G$. The diameter of $G$ denoted by $d(G)$, is the maximum distance between any pair of vertices http://dx.doi.org/10.29252/asta.4.1.51

MSC(2010): Primary: 05C50

Keywords: Distance spectrum, Distance energy, Line graphs

Received: 30 November 2017, Accepted: 07 March 2018

*Corresponding author

(C) 2017 Yazd University. 
of $G$ [3]. The eigenvalues of $D(G)$ are said to be the $D$ - eigenvalues of $G$ and form the $D-$ spectrum of $G$, denoted by $\operatorname{spec}_{D}(G)$.

The ordinary graph spectrum is formed by the eigenvalues of the adjacency matrix [5]. In what follows we denote the ordinary eigenvalues of the graph $G$ by $\lambda_{i}, i=1,2, \ldots, p$, and the respective spectrum by $\operatorname{spec}(G)$. Since the adjacency matrix is real symmetric, the eigenvalues are real and can be labelled so that $\lambda_{1} \geq \lambda_{2} \geq \cdots \geq \lambda_{p}$.

In a similar manner as the distance matrix is symmetric, all its eigenvalues $\mu_{i}, i=1,2, \ldots, p$, are real and can be labeled so that $\mu_{1} \geq \mu_{2} \geq \cdots \geq \mu_{p}$.

Two graphs $G$ and $H$ for which $\operatorname{spec}_{D}(G)=\operatorname{spec}_{D}(H)$ are said to be $D$ - cospectral. Otherwise, they are non-D- cospectral.

The $D$ - energy, $E_{D}(G)$, of $G$ [I2] is defined as

$$
E_{D}(G)=\sum_{i=1}^{p}\left|\mu_{i}\right| .
$$

Two graphs with equal $D$ - energy are said to be $D$ - equienergetic. $D$ - cospectral graphs are evidently D-equienergetic. Therefore, in what follows we focus our attention to $D$ - equienergetic non $-D-$ cospectral graphs.

The concept of $D$ - energy, Eq. (1), was introduced by Indulal et.al [12]. This definition was motivated by the much older [7] and nowadays extensively studied [9, 10, 11, 14, 15, 17, 18] graph energy, defined in a manner fully analogous to Eq. (1), but in terms of the ordinary graph eigenvalues (eigenvalues of the adjacency matrix, see [5]).

The characteristic polynomial of the $D$ - matrix and the corresponding spectra have been considered in [6, 8 ]. Moore and Moser in [16] showed for the first time that almost all graphs are of diameter 2 and this result was generalized by Bollobas in [2]. Thus a discussion of graphs of small diameter pertains to almost all graphs.

In [12] the distance spectrum of some graphs of diameter 2 and 3 are established. Also a lower bound for the largest eigenvalue of $D$, and bounds for the $D$ - energy are obtained. Some pairs of $D$ - equienergetic graphs of diameter 2 , on $p \equiv 1 \bmod (3)$ and $p \equiv 0 \bmod (6)$ vertices are also constructed. In [1]3], the distance spectra of some graphs obtained from cycles is derived and a pair of $D$ - equienergetic bipartite graphs on $24 t, t \geq 3$, vertices is also constructed.

In this paper we discuss the distance spectra of the complements of iterated line graphs of regular graphs and construct one more class of $D$ - equienergetic graphs. For some recent results see [I] and the papers cited there in.

A work of this type is reported here for the first time.

Let $G$ be a graph. Then its complement and line graph are respectively denoted by $\bar{G}$ and $L(G)[5]$. 
All graphs considered in this paper are simple, connected and we follow [5] for spectral graph theoretic terminology.

The considerations in the subsequent sections are based on the applications of the following.

Lemma 1.1. [5] Let $G$ be an $r$-regular graph. Then $r$ is a simple and the greatest eigenvalue of $G$.

Theorem 1.2. [5] Let $G$ be an $r$-regular graph on $p$ vertices. Then $\bar{G}$ is $p-r-1$ regular and $L(G)$ is $2 r-2$ regular. If $\left\{r, \lambda_{2}, \lambda_{3}, \ldots, \lambda_{p}\right\}$ are the adjacency eigenvalues of $G$, then

(1) The adjacency eigenvalues of $\bar{G}$ are $p-r-1$ and $-1-\lambda_{i}, i=2,3, \ldots, p$.

(2) The adjacency eigenvalues of $L(G)$ are $2 r-2, \lambda_{i}+r-2, i=2,3, \ldots, p$ and -2 with multiplicity $\frac{p(r-2)}{2}$.

Theorem 1.3. [IV] Let $G$ be an $r$ - regular graph of order $p$ and $d(G)=2$. If $\left\{r, \lambda_{2}, \lambda_{3}, \ldots, \lambda_{p}\right\}$ are its adjacency eigenvalues, then its $D$ - eigenvalues are $2 p-r-2$ and $-\left(\lambda_{i}+2\right), i=$ $2,3, \ldots, p$.

Lemma 1.4. [4] Let $G$ be an $r$ - regular graph on $p$ vertices. Let $p_{k}$ and $r_{k}$ denote the order and degree of its $k^{\text {th }}$ iterated line graph $L^{k}(G)$. Then

$$
\begin{aligned}
& p_{k}=\frac{p}{2^{k}} \prod_{i=0}^{k-1}\left(2^{i} r-2^{i+1}+2\right), \\
& r_{k}=2^{k} r-2^{k+1}+2 .
\end{aligned}
$$

\section{ON GRAPH COMPLEMENTS WITH DIAMETER 2}

In this section we prove the following theorem.

Theorem 2.1. Let $G$ be an $r-$ regular graph on $p$ vertices. If $r \leq \frac{p-1}{2}$, then $d(\bar{G})=2$.

Proof: Let $u$ and $v$ be two vertices of $G$. If $u$ not adjacent to $v$ in $G$, then $d(u, v)=1$ in $\bar{G}$. Now let $u$ adjacent to $v$ in $G$. Then $u$ and $v$ are collectively adjacent to atmost $2 r-2$ vertices other than themselves. Hence they are collectively adjacent to atmost $p-3$ vertices. Therefore always there exists a vertex $w$ in $G$ not adjacent to $u$ and $v$. Thus in $\bar{G}, w$ is adjacent to both $u$ and $v$ and hence $d(u, v)=2$. Hence the theorem follows.

Theorem 2.2. Let $G$ be an $r$ - regular graph on $p$ vertices with $r \leq \frac{p-1}{2}$. Then $d\left(\overline{L^{k}(G)}\right)=2$ for all $k \geq 1$.

Proof: Let $G$ be an $r$ - regular graph on $p$ vertices, $p \geq 8$. Let $p_{k}$ and $r_{k}$ respectively denote the order and regularity of the $k^{\text {th }}$ iterated line graph $L^{k}(G)$ of $G$. Then by Lemma 
ए.4

$$
\begin{aligned}
& p_{k}=\frac{p}{2^{k}} \prod_{i=0}^{k-1}\left(2^{i} r-2^{i+1}+2\right), \\
& r_{k}=2^{k} r-2^{k+1}+2 .
\end{aligned}
$$

Now we shall prove that

$$
p_{k}-1-2 r_{k} \geq 0
$$

for all $k \geq 1$ and $2 r \leq(p-1)$

We have

$$
\begin{aligned}
p_{k} & =\frac{p}{2^{k}} \prod_{i=1}^{k-1}\left(2^{i} r-2^{i+1}+2\right) \\
& =\frac{p}{2^{k-1}} \prod_{i=1}^{k-2}\left(2^{i} r-2^{i+1}+2\right) \frac{1}{2}\left(2^{k-1} r-2^{k}+2\right) \\
& =p_{k-1}\left(2^{k-2} r-2^{k-1}+1\right) . \\
r_{k} & =2^{k} r-2^{k+1}+2 . \\
p_{k}-1-2 r_{k} & =\left(p_{k-1}\left(2^{k-2} r-2^{k-1}+1\right)-1\right)-2\left(2^{k} r-2^{k+1}+2\right) \\
& =p_{k-1}\left(2^{k-2} r-2^{k-1}+1\right)-1-8\left(2^{k-2} r-2^{k-1}+1-\frac{1}{2}\right) \\
& =p_{k-1} t-1-8\left(t-\frac{1}{2}\right) \\
& =\left(p_{k-1}-8\right) t+3 \geq 0, \text { since } p_{k-1} \geq p \geq 8 \text { and } t=2^{k-2} r-2^{k-1}+1 \geq 0 .
\end{aligned}
$$

Thus $r_{k} \leq \frac{p_{k}-1}{2}$ and hence by Theorem [.], $d\left(\overline{L^{k}(G)}\right)=2$.

Lemma 2.3. Let $G$ be an $r$ - regular graph on $p$ vertices. Let $\left\{r, \lambda_{2}, \lambda_{3}, \ldots, \lambda_{p}\right\}$ are the adjacency eigenvalues of $G$. If $d(\bar{G})=2$, then the $D$ - eigenvalues of $\bar{G}$ are $\left\{p+r-1, \lambda_{2}-\right.$ $\left.1, \lambda_{3}-1, \ldots, \lambda_{p}-1\right\}$.

Proof: Lemma follows from Theorems ‥2 and ‥3.

Theorem 2.4. Let $G$ be an $r$ - regular graph on $p$ vertices with $r \leq \frac{p-1}{2}$. Then $E_{D}\left(\overline{L^{2}(G)}\right)=$ $3 \operatorname{pr}(r-2)$.

Proof: By Theorem ए.2, the adjacency eigenvalues of $L^{2}(G)$ are

$$
\left(\begin{array}{cccccc}
4 r-6 & \lambda_{2}+3 r-6 & \ldots & \lambda_{p}+3 r-6 & 2 r-6 & -2 \\
1 & 1 & \ldots & 1 & \frac{p(r-2)}{2} & \frac{p r(r-2)}{2}
\end{array}\right)
$$


Now by Theorem [2.2, $d\left(\overline{L^{2}(G)}\right)=2$. Then by Lemma [2.3], the $D$ - eigenvalues of $\overline{L^{2}(G)}$ are

$$
\left(\begin{array}{cccccc}
\frac{p r(r-1)}{2}+4 r-7 & \lambda_{2}+3 r-7 & \ldots & \lambda_{p}+3 r-7 & 2 r-7 & -3 \\
1 & 1 & \ldots & 1 & \frac{p(r-2)}{2} & \frac{p r(r-2)}{2}
\end{array}\right) .
$$

Now by the ordering of eigenvalues $\lambda_{i}$ as $r=\lambda_{1} \geq \lambda_{2} \geq \cdots \geq \lambda_{p}$, we have for $r \geq 4$, the only negative $D$ - eigenvalue of $\overline{L^{2}(G)}$ is -3 with multiplicity $\frac{p r(r-2)}{2}$. Thus $E_{D}\left(\overline{L^{2}(G)}\right)=3 p r(r-2)$.

Corollary 1. Let $G$ be an $r-$ regular graph on $p$ vertices with with $r \leq \frac{p-1}{2}$. Let $p_{k}$ and $r_{k}$ denote the order and degree of its $k^{\text {th }}$ iterated line graph $L^{k}(G)$. Then

$$
E_{D}\left(\overline{L^{k}(G)}\right)=3 p(r-2)\left(2^{k-2} r-2^{k-1}+2\right) \prod_{i=0}^{k-3}\left(2^{i} r-2^{i+1}+2\right) .
$$

\section{Proof:}

Since $L^{k}(G)=L^{2}\left(L^{k-2}(G)\right)$, from Theorem 2.4 we have

$$
E_{D}\left(\overline{L^{k}(G)}\right)=3 p_{k-2} r_{k-2}\left(r_{k-2}-2\right), \quad k \geq 3 .
$$

Now by Lemma प.4

$$
p_{k-2}=\frac{p}{2^{k-2}} \prod_{i=0}^{k-3}\left(2^{i} r-2^{i+1}+2\right) r_{k-2}=2^{k-2} r-2^{k-1}+2 .
$$

Now substituting Equation [ in Equation 3 we obtain

$$
E_{D}\left(\overline{L^{k}(G)}\right)=3 p(r-2)\left(2^{k-2} r-2^{k-1}+2\right) \prod_{i=0}^{k-3}\left(2^{i} r-2^{i+1}+2\right) .
$$

Theorem 2.5. Let $G_{1}$ and $G_{2}$ be two non $D$-cospectral regular graphs of the same order $p$ and of the same degree $r$. Let $2 r \leq p-1$. Then for any $k \geq 2, r \geq 4$, the graphs $\overline{L^{k}\left(G_{1}\right)}$ and $\overline{L^{k}\left(G_{2}\right)}$ form a pair of non D-cospectral, D- equienergetic graphs of equal order and of equal number of edges with energy $3 p(r-2)\left(2^{k-2} r-2^{k-1}+2\right) \prod_{i=0}^{k-3}\left(2^{i} r-2^{i+1}+2\right)$.

\section{REFERENCES}

[1] M. Aouchiche and P. Hansen, Distance spectra of graphs: A survey, Lin. Algebra Appl. 458 (2014), 301-386.

[2] B. Bollobas, The diameter of random graphs, Trans. Amer. Math. Soc. 267 (1981), 41-52.

[3] F. Buckley and F. Harary, Distance in Graphs, Addison Wesley, Redwood. 1990.

[4] F. Buckley, The size of iterated line graphs, Graph Theory Notes New York. 25 (1993), 33-36.

[5] D.M. Cvetkovic, M. Doob and H. Sachs, Spectra of Graphs-Theory and Applications, Academic Press, (1980). 
[6] M. Edelberg, M.R. Garey and R.L. Graham, On the distance matrix of a tree, Discrete Math. 14 (1976), 23-39.

[7] F.R. Gantmacher, Applications of the Theory of Matrices (Interscience, New York, 1959), Google Schola. (1969), p.64.

[8] R.L. Graham and L. Lovasz, Distance matrix polynomials of trees. Adv Math. 29 (1978), 60-88.

[9] I. Gutman, On graphs whose energy exceeds the number of vertices, Lin. Algebra Appl. 429 (11-12) (2008), 2670-2677.

[10] I. Gutman, S. Zare Firoozabadi, J.A. delaPena and J. Rada, On the energy of regular graphs, Match Commun. Math. Comput. Chem. 57 (2007), 435-442.

[11] W.H. Haemers, Strongly regular graphs with maximal energy, Lin. Algebra Appl. 429 (11-12) (2008), 2719-2723.

[12] G. Indulal, I. Gutman and A. Vijayakumar, On distance energy of graphs, Match Commun. Math. Comput. Chem. 60 (2008), 461- 472.

[13] G. Indulal and I. Gutman, On the distance spectra of somegraphs, Math. Commun. 13 (2008), 123-131.

[14] G. Indulal and A. Vijayakumar, A note on energy of some graphs, Match Commun. Math. Comput. Chem. 59 (2008), 269-274.

[15] X. Li and J. Zhang, On bicyclic graphs with maximal energy, Lin. Algebra Appl. 427 (2007), $87-98$.

[16] J.W. Moon and L. Moser, Almost all $(0,1)$ matrices are primitive, Studia Sci Math Hungar. 1 (1966), 153-156.

[17] V. Nikiforov, The energy of graphs and matrices, J. Math. Anal. Appl. 326 (2007), 1472-1475.

[18] I. Shparlinski, On the energy of some circulant graphs, Lin. Algebra Appl. 414 (2006), 378382.

\section{Gopalapillai Indulal}

Department of Mathematics

St.Aloysius College, Edathua

Alappuzha , India - 689573

indulalgopal@gmail.com 